\title{
Jurassic: A chemically anomalous structure in the Galactic halo*
}

\author{
José G. Fernández-Trincado ${ }^{1,2}$, Timothy C. Beers ${ }^{3}$, and Dante Minniti ${ }^{4,5}$ \\ 1 Institut Utinam, CNRS-UMR 6213, Université Bourgogne-Franche-Comté, OSU THETA Franche-Comté, \\ Observatoire de Besançon, BP 1615, 251010 Besançon Cedex, France \\ e-mail: jfernandez@obs-besancon.fr \\ 2 Instituto de Astronomía y Ciencias Planetarias, Universidad de Atacama, Copayapu 485, Copiapó, Chile \\ e-mail: jose.fernandez@uda.cl \\ 3 Department of Physics and JINA Center for the Evolution of the Elements, University of Notre Dame, Notre Dame, \\ IN 46556, USA \\ ${ }^{4}$ Depto. de Cs. Físicas, Facultad de Ciencias Exactas, Universidad Andrés Bello, Av. Fernández Concha 700, Las Condes, \\ Santiago, Chile \\ 5 Vatican Observatory, 00120 Vatican City State, Italy
}

Received 14 September 2020 / Accepted 20 October 2020

\begin{abstract}
Detailed elemental-abundance patterns of giant stars in the Galactic halo measured by the Apache Point Observatory Galactic Evolution Experiment (APOGEE-2) have revealed the existence of a unique and significant stellar subpopulation of silicon-enhanced $([\mathrm{Si} / \mathrm{Fe}] \gtrsim+0.5)$ metal-poor stars, spanning a wide range of metallicities $(-1.5 \lesssim[\mathrm{Fe} / \mathrm{H}] \lesssim-0.8)$. Stars with over-abundances in $[\mathrm{Si} / \mathrm{Fe}]$ are of great interest because these have very strong silicon $\left({ }^{28} \mathrm{Si}\right)$ spectral features for stars of their metallicity and evolutionary stage, offering clues about rare nucleosynthetic pathways in globular clusters (GCs). Si-rich field stars have been conjectured to have been evaporated from GCs, however, the origin of their abundances remains unclear, and several scenarios have been offered to explain the anomalous abundance ratios. These include the hypothesis that some of them were born from a cloud of gas previously polluted by a progenitor that underwent a specific and peculiar nucleosynthesis event or, alternatively, that they were due to mass transfer from a previous evolved companion. However, those scenarios do not simultaneously explain the wide gamut of chemical species that are found in Si-rich stars. Instead, we show that the present inventory of such unusual stars, as well as their relation to known halo substructures (including the in situ halo, Gaia-Enceladus, the Helmi Stream(s), and Sequoia, among others), is still incomplete. We report the chemical abundances of the iron-peak $(\mathrm{Fe})$, the light- $(\mathrm{C}$ and $\mathrm{N})$, the $\alpha-(\mathrm{O}$ and $\mathrm{Mg})$, the odd- $Z(\mathrm{Na}$ and $\mathrm{Al})$, and the $s$-process (Ce and Nd) elements of 55 newly identified Si-rich field stars (among more than 600000 APOGEE-2 targets), which exhibit over-abundances of $[\mathrm{Si} / \mathrm{Fe}]$ as extreme as those observed in some Galactic GCs, and they are relatively well distinguished from other stars in the $[\mathrm{Si} / \mathrm{Fe}]-[\mathrm{Fe} / \mathrm{H}]$ plane. This new census confirms the presence of a statistically significant and chemically-anomalous structure in the inner halo: Jurassic. The chemo-dynamical properties of the Jurassic structure is consistent with it being the tidally disrupted remains of GCs, which are easily distinguished by an over-abundance of $[\mathrm{Si} / \mathrm{Fe}]$ among Milky Way populations or satellites.
\end{abstract}

Key words. Galaxy: structure - Galaxy: kinematics and dynamics - stars: abundances - stars: chemically peculiar globular clusters: general - techniques: spectroscopic

\section{Introduction}

The stellar content of the halo of the Milky Way (MW) is littered by a mixture of stellar debris of completely and/or partially destroyed dwarf galaxies and globular clusters (GCs) (e.g., Helmi et al. 1999; Carollo et al. 2007, 2010; Nissen \& Schuster 2010; Fernández-Trincado et al. 2013, 2015a,b, 2016a,b, 2017, 2019a,b,c, 2020a,b,c; Recio-Blanco et al. 2017; Bekki 2019; Koch et al. 2019; Massari et al. 2019; Hanke et al. 2020; Thomas et al. 2020; Yuan et al. 2020; Wan et al. 2020; Naidu et al. 2020), which preserve signatures of the Galaxy's assembly history (see Naidu et al. 2020, for a recent review).

Most of the stellar halo debris identified to date extend out to several hundred kiloparsecs, and they have been identified in a wide variety of discrete structures, preserving important insight into the earliest accretion events. Gaia Data Release 2 (DR2; Gaia Collaboration 2018), complemented with ground-

\footnotetext{
* Tables A.1-A.4 are only available at the CDS via anonymous ftp to cdsarc.u-strasbg. fr (130.79.128.5) or via http://cdsarc. u-strasbg.fr/viz-bin/cat/J/A+A/644/A83
}

based spectroscopic surveys, has provided compelling evidence that both the inner- and outer-halo populations of the MW are dominated by an ex-situ formation scenario being built up through massive $\left(>10^{10} M_{\odot}\right)$ accretion events that have probably occurred $\sim 8-11$ Gyr ago, including the Gaia-Enceladus (G-E) dwarf galaxy (Belokurov et al. 2018; Haywood et al. 2018; Helmi et al. 2018; Koppelman et al. 2018; Myeong et al. 2018) and accompanied by other significant merger events such as Sequoia (Myeong et al. 2019), the Helmi stream(s) (Helmi et al. 1999; Chiba \& Beers 2000; Koppelman et al. 2019a), Kraken (Kruijssen et al. 2019), Thamnos 1 \& 2 (Koppelman et al. 2019b), the disrupting Sagittarius dwarf galaxy (Ibata et al. 1994; Law \& Majewski 2010; Majewski et al. 2013; Hasselquist et al. 2019; Hayes et al. 2020), as well as streams recently identified as debris from very metal-poor globular clusters (Thomas et al. 2020; Wan et al. 2020; Yuan et al. 2020), and the Fimbul thul structure associated with the unusual globular cluster $\omega$ Cen (Ibata et al. 2019). In this paper, we add a chemically anomalous halo population of Si-rich stars to this collection (Fernández-Trincado et al. 2019c), which we refer to as the Jurassic structure. 
There also exists a wealth of observational evidence for a possible in situ channel, especially for the inner-halo population itself, which is spatially, kinematically, and chemically distinguishable from the outer-halo population (Carollo et al. 2007, 2010; Beers et al. 2012; An et al. 2013, 2015; An \& Beers 2020) and thought to have formed, in part, from gas accreted by the MW at early times (Carollo et al. 2013; Tissera et al. 2014; Hawkins et al. 2015; Hayes et al. 2018; Fernández-Alvar et al. 2018, 2019). These studies illustrate the complex formation history of the stellar halo of the MW, which may involve a mixture of stars that likely formed in situ and stellar debris, which were accreted from different structures.

Although the morphology and chemo-dynamical properties of the stellar halo have been extensively explored over the past few decades (see Beers \& Christlieb 2005; Ivezić et al. 2012; Helmi 2020 for reviews), a long-standing problem remains as to how the different formation scenarios for the stellar halo may be discriminated from one another. Current large stellar spectroscopic surveys of the MW, such as the Apache Point Observatory Galactic Evolution Experiment (APOGEE: Majewski et al 2017), enable methods, such as chemical tagging which is based on the principle that the chemical composition of stars reflect the site of their formation (Freeman \& Bland-Hawthorn 2002; Feltzing \& Chiba 2013; Ting et al. 2015; Hogg et al. 2016), can help clarify the picture of where the majority of halo stars are produced and what caused them to achieve their current physical properties.

The search for field stars that were born in GCs (Lind et al. 2015; Martell et al. 2016; Fernández-Trincado et al. 2015b, 2016a,b, 2017, 2019a,b,c,d, 2020a; Simpson et al. 2020) is one clear example of the power of chemical tagging. This is possible because GCs appear to be the only environment responsible for the presence of light-element anti-correlations at all stellar evolutionary phases (e.g., Martell et al. 2016; Pancino et al. 2017; Bastian \& Lardo 2018; Masseron et al. 2019; Mészáros et al. 2020), unless they are part of a binary system (Bastian \& Lardo 2018; Fernández-Trincado et al. 2019a), or part of the new kind of recently discovered anomalous Phosphorus-rich field stars (see, e.g., Masseron et al. 2020). Thus, a complete census of all those chemically anomalous stars will help develop a better understanding for the assembly of the inner and outer halo, where substantial amount of stellar debris from GCs are thought to currently reside (see, e.g., Martell et al. 2016; FernándezTrincado et al. 2019b,c, 2020a).

In this paper, we update the census of silicon-enriched metalpoor stars, making use of data from the APOGEE-2 survey (Majewski et al. 2017). The silicon-enriched metal-poor stars are of particular interest as they belong to the exclusive collection of metal-poor stars in GCs where ${ }^{28} \mathrm{Si}$ leaking from the Mg-Al cycle at high temperature (see e.g., Mészáros et al. 2015, 2020). Thus, such a search in other Galactic environments provides strong evidence either for or against the uniqueness of the progenitor stars to GCs evolution. This paper is outlined as follows. In Sect. 2, we present details and information regarding the APOGEE-2 data set and the incremental data used in this work. In Sect. 3, we describe our sample selection, the adopted stellar parameters, and the methodology employed to determine the elemental abundances. In Sect. 5, we present the newly identified Si-rich stars. In Sect. 6, we present the statistical significance of the Jurassic structure. In Sect. 7, we present details regarding the elemental abundances of the stars in the Jurassic structure. In Sect. 8, we present a dynamical study of the stars in the Jurassic structure. Our conclusions are drawn in Sect. 9.

\section{The APOGEE $\boldsymbol{H}$-band spectroscopic survey}

The dataset analyzed in this work comes primarly from the Apache Point Observatory Galactic Evolution Experiment (APOGEE: Zasowski et al. 2013; Majewski et al. 2017) and its successor APOGEE-2 (Zasowski et al. 2017), which have collected high-resolution $(R \sim 22500) H$-band spectra (near-IR, $\sim 15145 \AA$ to $16960 \AA$, vacuum wavelengths) for almost 430000 sources in their sixteenth data release (DR16, Ahumada et al. 2020), as part of the Sloan Digital Sky Survey IV (Blanton et al. 2017). Here we take advantage of new data taken subsequent to the DR16 release, which were reduced with the same pipeline as the DR16 stars. This new incremental data set provides to the scientific community spectra of more than 680000 stars; we refer to these data as the incremental APOGEE-2 DR16 plus (hereafter APOGEE-2+)

APOGEE-2+ includes data taken from both the Northern and Southern hemisphere using the APOGEE-2 spectrographs (Eisenstein et al. 2011; Wilson et al. 2012, 2019) mounted on the $2.5 \mathrm{~m}$ Sloan Foundation telescope (Gunn et al. 2006) at Apache Point Observatory in New Mexico (APOGEE-2N: North, APO), and in the $2.5 \mathrm{~m}$ Irénée du Pont telescope (Bowen \& Vaughan 1973) at Las Campanas Observatory (APOGEE-2S: South, LCO) in Chile. For details regarding the APOGEE atmosphericparameter analysis we direct the reader to the description of the APOGEE Stellar Parameter and Chemical Abundances pipeline (ASPCAP: García Pérez et al. 2016), while for details about the grid of synthetic spectra and errors see Holtzman et al. (2015, 2018) and Jönsson et al. (2018, 2020). We also refer the reader to Nidever et al. (2015) for further details regarding the data reduction pipeline for APOGEE2+. The model grids for APOGEE$2+$ are based on a complete set of MARCS (Gustafsson et al. 2008) stellar atmospheres, which now extend to effective temperatures as low as $3200 \mathrm{~K}$, and spectral synthesis using the Turbospectrum code (Plez 2012).

\section{Data}

Since we are primarily interested in the detection and mapping of Si-rich metal-poor stars, our focus in this work is on giants in the metallicity range between $[\mathrm{Fe} / \mathrm{H}]=-1.8$ and -0.7 . The Si-rich stars were first discovered in Fernández-Trincado et al. (2019c), and were hypothesized to belong to a new sub-population of the inner stellar halo. Here, we conduct a large search for such stars in the APOGEE-2+ catalog. By imposing a lower limit on metallicity, $[\mathrm{Fe} / \mathrm{H}]>-1.8$, we include stars with high-quality spectra and reliable parameters and abundances. The requirement of an upper limit of $[\mathrm{Fe} / \mathrm{H}]<-0.7$ minimizes the presence of stars belonging to the disk system. Thus, we selected a sample of giant stars, adopting conservative cuts on the columns of the APOGEE-2+ catalogue in the following way:

1. $S / N>60$ pixel $^{-1}$. This cut was chosen to ensure that we are selecting spectra that have well-known uncertainties in their stellar parameters and chemical abundances, and remove stars with lower quality spectra (e.g., García Pérez et al. 2016).

2. $3200 \mathrm{~K}<T_{\mathrm{eff}}^{\mathrm{ASPAP}}<6000 \mathrm{~K}$. This temperature range ensures that the stellar parameters are reliably and consistently determined, and maximizes the overall quality of the abundances considered (García Pérez et al. 2016; Holtzman et al. 2018).

3. The estimated $\log g^{\mathrm{ASPCAP}}$ must be less than 3.6. This cut was chosen to ensure that stars have more accurate ASPCAPderived parameters than the stars with $\log g^{\mathrm{ASPCAP}}>3.6$. Due to the lack of asteroseismic surface gravities for dwarfs, 
only stars with $\log g<3.6$ have calibrated surface gravity estimates (see e.g., Jönsson et al. 2018, 2020).

4. $A S P C A P F L A G==0$. This cut ensures that there were no major flagged issues, that is, low signal-to-noise, poor synthetic spectral fit, stellar parameters near grid boundaries, among others (e.g., Holtzman et al. 2015; García Pérez et al. 2016).

5. For our selection, we also disregarded stars in GCs from our sample, i.e., those sources analyzed in Masseron et al. (2019) and Mészáros et al. (2020).

6. Lastly, field stars identified previously as P-, N- and Si-rich stars from Martell et al. (2016), Fernández-Trincado et al. (2016b, 2017, 2019a,b,c,d), and Masseron et al. (2020) were excluded from our sample.

Our initial sample contains about 19700 stars, which is four times larger than the sample of Fernández-Trincado et al. (2019c) analyzed in previous data releases. After the internal release of the APOGEE-2+ catalogue, we discovered many more stars belonging to the Si-rich sample, which we report here as part of a larger homogeneous census. We have added these new stars to the sample, derived new atmospheric parameters, and ran our abundance determination pipeline BACCHUS (Masseron et al. 2016) with the same setup used in Fernández-Trincado et al. (2019c). Here, we proceed with a detailed re-analysis of the newly discovered Si-rich stars with the BACCHUS code, as ASPCAP introduce its own set of problems for the effective temperatures and $[\mathrm{X} / \mathrm{Fe}]$ at low metallicities, $[\mathrm{Fe} / \mathrm{H}]<-0.7 \mathrm{dex}$ (see, e.g., Fernández-Trincado et al. 2019a,b,c, 2020b; Nataf et al. 2019; Mészáros et al. 2015, 2020).

\section{Stellar parameters and abundance determinations}

Since the method of deriving atmospheric parameters and abundances is identical to that as described in Fernández-Trincado et al. (2019c), we only provide a short overview of it in this paper. As before (Fernández-Trincado et al. 2016b, 2017, 2019a,b,c,d), we use the uncalibrated stellar parameters for $T_{\text {eff }}^{\text {ASPCAP }}$ (FPARAM_1) and $\log g^{\text {ASPCAP }}$ (FPARAM_2). First, we made a careful inspection of each $H$-band spectrum with the BACCHUS code to derive the metallicity, broadening parameters, and chemical abundances, based on a line-by-line approach in the same manner as in Fernández-Trincado et al. (2019c), and summarized here for guidance. The BACCHUS code relies on the radiative transfer code Turbospectrum (Alvarez \& Plez 1998; Plez 2012) and the MARCS model atmosphere grid (Gustafsson et al. 2008).

For each element and each line, the abundance determination proceeds as in Hawkins et al. (2016) and FernándezTrincado et al. (2019b): (a) A spectrum synthesis, using the full set of atomic and molecular line lists described in Shetrone et al. (2015), (Neodymium: Nd II) (Hasselquist et al. 2016) and (Cerium: Ce II) (Cunha et al. 2017) (this set of lists is internally labeled as linelist.20170418 based on the date of creation in the format YYYYMMDD). This is used to find the local continuum level via a linear fit; (b) Cosmic and telluric rejections are performed; (c) The local $\mathrm{S} / \mathrm{N}$ is estimated; (d) A series of flux points contributing to a given absorption line is automatically selected; and (e) Abundances are then derived by comparing the observed spectrum with a set of convolved synthetic spectra characterized by different abundances. Then, four different abundance determination methods are used: (1) line-profile fitting; (2) core line-intensity comparison; (3) global goodnessof-fit estimate; and (4) equivalent-width comparison. Each diag- nostic yields validation flags. Based on these flags, a decision tree then rejects or accepts each estimate, keeping the best-fit abundance. Here, we adopted the $\chi^{2}$ diagnostic as the abundance because it is the most robust. However, we store the information from the other diagnostics, including the standard deviation between all four methods.

A mix of heavily $\mathrm{CN}$-cycled and $\alpha$-poor MARCS models were used, as well as the same molecular lines adopted by Smith et al. (2013), and employed to determine the C, N, and O abundances. In addition, we have adopted the $\mathrm{C}, \mathrm{N}$, and $\mathrm{O}$ abundances that satisfy the fitting of all molecular lines consistently; i.e., we first derive ${ }^{16} \mathrm{O}$ abundances from ${ }^{16} \mathrm{OH}$ lines, then derive ${ }^{12} \mathrm{C}$ from ${ }^{12} \mathrm{C}^{16} \mathrm{O}$ lines, and ${ }^{14} \mathrm{~N}$ from ${ }^{12} \mathrm{C}^{14} \mathrm{~N}$ lines; the $\mathrm{CNO}$ abundances are derived several times to minimize the $\mathrm{OH}, \mathrm{CO}$, and $\mathrm{CN}$ dependences (see, e.g., Smith et al. 2013; Fernández-Trincado et al. 2016b, 2017, 2019a,b,c,d, 2020d).

Lastly, to provide a consistent chemical analysis, we redetermine the chemical abundances, assuming as input the uncalibrated effective temperature ( $T_{\mathrm{eff}}^{\mathrm{ASPCAP}}$ or FPARAM_1), surface gravity ( $\log g^{\mathrm{ASPCAP}}$ or FPARAM_2), and metallicity $([\mathrm{M} / \mathrm{H}]$ or FPARAM_3) as derived by ASPCAP/APOGEE-2+ run. We also applied a simple approach of fixing $T_{\mathrm{eff}}^{\text {pho }}$ and $\log g$ to values determined independently of spectroscopy, in order to check for any significant deviation in the chemical abundances. For this, the photometric effective temperatures were calculated from the $J_{2 \text { MASS }}-K_{s, 2 \text { MASS }}$ color relation using the methodology presented in González Hernández \& Bonifacio (2009).

Photometry is extinction corrected using the Rayleigh Jeans Color Excess (RJCE) method (Majewski et al. 2011). We estimate surface gravity from 10 Gyr PARSEC (Bressan et al. 2012) isochrones, as $10 \mathrm{Gyr}$ is the typical age of Galactic GCs (see, e.g., Baumgardt et al. 2019); here we assume that the stars in the Jurassic structure could be possible GC migrants.

The adopted stellar parameters are listed in Table A.1. It is also important to note that the absence of radial-velocity variation, as listed in the same table (visit-to-visit variation, $\mathrm{RV}_{\text {SCATTER }}<2 \mathrm{~km} \mathrm{~s}^{-1}$ ), does not support any evidence for a binary companion, i.e., none of the newly identified Si-rich giants in the Jurassic structure has a strong variability in its radial velocity over the period of the APOGEE-2+ observations ( $\$ 6$ months). However, long-term radial-velocity monitoring of all of our stars would naturally be the best course to establish the number of such objects formed through the binary channel.

Figure 1 compares the sensitivity to the derived atmospheric parameters, depending on the species and line in question. When the spectroscopic and photometry-based atmospheric parameters were adopted, we found large discrepancies in the effective temperature $(\gtrsim 200 \mathrm{~K})$ and surface gravity $(\gtrsim 0.6 \mathrm{dex})$ for a few stars, particularly for hotter $(\gtrsim 5000 \mathrm{~K})$ stars. This issue does not strongly affect the derived $[\mathrm{Si} / \mathrm{Fe}]$ abundance ratios, but other chemical species such as nitrogen, oxygen, aluminum, cerium, and neodymium are more $(\gtrsim 0.2 \mathrm{dex})$ affected by these atmospheric discrepancies. However, as can be seen in the same figure, these large discrepancies do not have a strong impact on our determined $[\mathrm{Si} / \mathrm{Fe}]$ abundance ratios, whose differences are $<0.07$ dex, much less that the reported intrinsic error.

Figure 2 confirms the reliability in the detected Si I lines, where the spectra of some Si-rich and Si-normal stars are compared in the relevant wavelength intervals. The Si-rich stars have remarkably stronger Sil lines which, in view of the similarity between the pairs of stars in all the other relevant parameters, can only mean that they have much higher silicon abundances. 


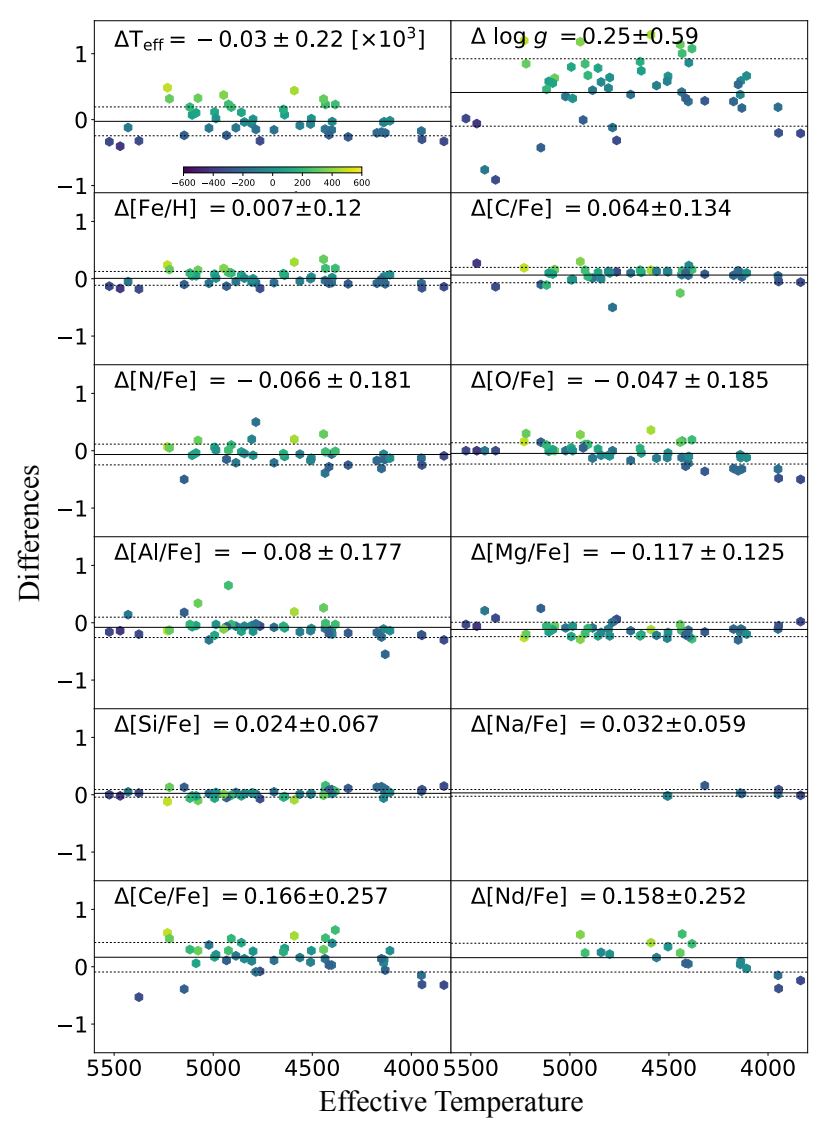

Fig. 1. Newly identified stars in the Jurassic structure analyzed in this work. Differences in atmospheric parameters and elemental abundances produced by two runs adopting different effective temperatures $\left(T_{\text {eff }}\right)$ and surface gravities $(\log g)$ : photometric versus uncalibrated ASPCAP values. The symbols are color-coded by the differences between the photometric and uncalibrated ASPCAP temperatures. The average and \pm errors (the standard deviation around the mean of the differences) are listed in the title of each panel.

Figure 1 also indicates that the BACCHUS code recovers the $[\mathrm{Fe} / \mathrm{H}]$ abundance ratios of these stars within $\sim 0.12$ dex for both the adopted photometric and spectroscopic temperatures. The adoption of a purely photometric temperature scale enables us to be somewhat independent of the ASPCAP pipeline, which provides important comparison data for future pipeline validation. However, as pointed out by Mészáros et al. (2020), the use of photometric temperatures introduces their own set of potential problems related to high $E(B-V)$ values, as the González Hernández \& Bonifacio (2009) relations are very sensitive to small changes in $E(B-V)$.

Table A.1 shows that $\sim 20 \%$ of the stars in the Jurassic structure have $E(B-V)>0.4$, so either the reddening and/or the photometric temperatures are not reliable. For this reason, we limit our discussion to stars in the Jurassic structure with abundance determinations from spectroscopic atmospheric parameters, similar to our previous papers. The complete set of abundances for ten chemical species - C, N, O, Na, Mg, Al, Si, $\mathrm{Fe}, \mathrm{Ce}$, and $\mathrm{Nd}-$ can be found in Table A.2.

Table A.3 list an example of the typical uncertainties for twenty six randomly selected stars in our sample, defined as:

$\sigma_{\text {total }}^{2}=\sigma_{[\mathrm{X} / \mathrm{H}], \mathrm{T}_{\mathrm{eff}}}^{2}+\sigma_{[\mathrm{X} / \mathrm{H}], \log g}^{2}+\sigma_{[\mathrm{X} / \mathrm{H}], \xi_{\mathrm{t}}}^{2}+\sigma_{\text {mean }}^{2}$

where $\sigma_{\text {mean }}^{2}$ is calculated using the standard deviation derived from the different abundances of the different lines for each element. The values of $\sigma_{[\mathrm{X} / \mathrm{H}], \mathrm{T}_{\mathrm{eff}}}^{2}, \sigma_{[\mathrm{X} / \mathrm{H}], \log g}^{2}$, and $\sigma_{[\mathrm{X} / \mathrm{H}], \xi_{\mathrm{t}}}^{2}$ are derived for the elements in each star using the sensitivity values of $\pm 100 \mathrm{~K}$ for the temperature, \pm 0.3 dex for $\log g$, and $0.05 \mathrm{~km} \mathrm{~s}^{-1}$ for the microturbulent velocity $\left(\xi_{\mathrm{t}}\right)$.

It is important to note that our results are compared with $[\mathrm{X} / \mathrm{Fe}]$ abundance ratios determined with the ASPCAP, thus, in order to proceed with an appropriate comparison we correct the ASPCAP by the typical offset of each chemical species between the BACCHUS and ASPCAP pipeline found for a control sample of $\sim 1000$ metal-poor $(-1.8 \lesssim[\mathrm{Fe} / \mathrm{H}] \lesssim-0.7)$ stars belonging to the main components of the MW (halo, disk, and bulge). At the same manner as in Fernández-Trincado et al. (2020b), we find that ASPCAP significantly underestimates most of the chemical species by about $\sim 0.1$ to 0.3 dex for most of the metal-poor stars (see also Nataf et al. 2019). Such offsets were taken into consideration for the whole MW stars from ASPCAP determinations.

\section{Stars in the Jurassic structure}

Figure 3 shows $[\mathrm{Si} / \mathrm{Fe}]$ versus $[\mathrm{Fe} / \mathrm{H}]$, as derived from the ASPCAP pipeline, for our initial sample. The overall behaviours for $[\mathrm{Si} / \mathrm{Fe}]$ as a function of $[\mathrm{Fe} / \mathrm{H}]$ shows a smooth transition between the thick-disk and halo population with low-[Si/Fe] $(\lesssim+0.5)$ abundance ratios, which we refer to as Si-normal stars in the main MW.

Our search for Si-rich stars begins with a silicon- and metallicity-based selection criterion in the same manner as in Fernández-Trincado et al. (2019c). Using over 19700 stars, we determined the boundary between the Si-rich and Si-normal field stars by identifying the trough in the $[\mathrm{Si} / \mathrm{Fe}]$ distribution in twelve metallicity bins. Figure 3 shows the boundary (light green thick lines) and the number of stars in each of the metallicity bins used to determine the separation between the Si-rich and the Si-normal sequences. The boundary between these two sequences was determined by estimating the average and the standard deviation in $[\mathrm{Si} / \mathrm{Fe}]$ per metallicity bin in the main body, i.e., the light green thick lines in Fig. 3 should be understood as $\langle[\mathrm{Si} / \mathrm{Fe}]\rangle_{\text {bin }}+3 \sigma_{[\mathrm{Si} / \mathrm{Fe}]}$ per metallicity bin. The bin sizes were chosen to ensure at least 400 stars were in each bin, with bin centres at $[\mathrm{Fe} / \mathrm{H}]=-1.75$ to -0.75 , in steps of 0.09 dex (the number of stars per metallicity bin are shown at the bottom in the same figure). We then label all stars with silicon over-abundances more than $+3 \sigma_{[\mathrm{Si} / \mathrm{Fe}]}$ above the $\langle[\mathrm{Si} / \mathrm{Fe}]\rangle_{\text {bin }}$ at fixed metallicity as Si-rich, which is the same as the selection in Fernández-Trincado et al. (2019c). This returns 55 stars as potential Si-rich stars relative to the final data set, which we have called the Jurassic structure.

Our first selection was simply based on ASPCAP abundances, however, for several other issues that might affect the abundance determinations in the metal-poor regime (see e.g., Jönsson et al. 2018), we have decided to adopt a detailed manual examination and visual inspection, in order to ensure that the spectral fit was adequate for those stars by using the Brussels Automatic Stellar Parameter (BACCHUS) code (Masseron et al. 2016) to rederive the chemical abundances of our sample, by adopting a simple line-by-line approach of selected atomic and molecule lines, under the assumption of local thermodynamic equilibrium (LTE), and using the standard iron ionization - excitation equilibrium technique. If the lines were not well-reproduced by the synthesis, or the lines were strongly blended or too weak in the spectra of stars to deliver reliable $[\mathrm{Si} / \mathrm{Fe}]$ ratios, they were rejected. Thus, the $[\mathrm{X} / \mathrm{Fe}]$ abundance ratios relies on abundance determinations from the BACCHUS pipeline, and $[\mathrm{Fe} / \mathrm{H}]$ has been determined from $\mathrm{Fe} I$ lines. 


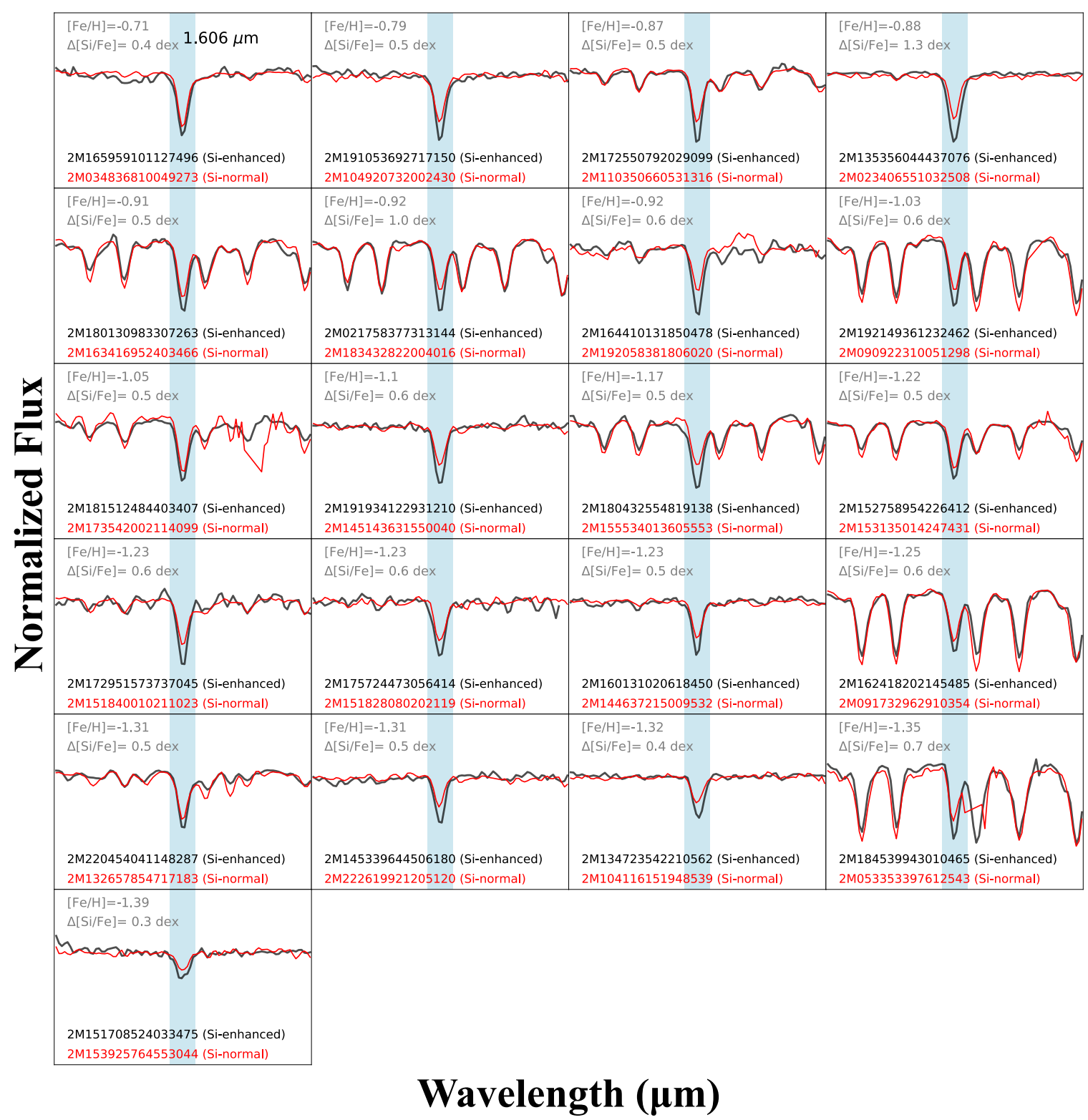

Fig. 2. Portions of 21 Si-rich stars (black) compared to Si-normal (red) stars, with similar stellar parameters, around the Si I absorption line at $1.606 \mu \mathrm{m}$ (blue shadow region). The stars are ordered by decreasing metallicity, which is indicated in the top left corner in each panel. The $\Delta[\mathrm{Si} / \mathrm{Fe}]$ between both stars is also shown.

\section{Statistical significance}

Fernández-Trincado et al. (2019c) already had some indication for the existence of a distinct stellar sub-population in the inner halo of the MW, which is separated relatively cleanly in the $[\mathrm{Al} / \mathrm{Fe}]-[\mathrm{Si} / \mathrm{Fe}]$ plane as shown in their Fig. 2. This new sub-population was proven to be statistically significant and to belong to a true low-density valley separating the Si-normal population from the Si-rich population, which exceeds the background level by a factor of $\sim 4$.

With our large sample, we revisit the statistical significance of the Jurasicc structure over a wide range of metallicities for an unprecedented homogeneous dataset, together with other previously identified Si-rich stars from the literature and highlighted as black filled "star" symbols in Fig. 4. There are also one Nrich star (TYC 5619-109-1, a possible early-AGB star) analyzed in Fernández-Trincado et al. (2016b) and Pereira et al. (2017); one N-rich bulge giant from Schiavon et al. (2017); one N-/Al- enhanced giant from Fernández-Trincado et al. (2017); four Nrich giants from Fernández-Trincado et al. (2019b); and eleven Si-/Al-enhanced giants from Fernández-Trincado et al. (2019c). All of them (with the exception of one star from Schiavon's sample and a few N-rich stars from the Fernández-Trincado's study) exhibit typical enhancement in $\mathrm{Al}$ (e.g., $[\mathrm{Al} / \mathrm{Fe}]>+0.5$ ), wellabove the typical Galactic levels.

We note that in Fernández-Trincado et al. (2019c) we searched for Si-stars exclusively enriched in $\mathrm{Al}([\mathrm{Al} / \mathrm{Fe}] \gtrsim+0.5)$, as aluminum appears to be one of the most effective chemical tags for GC-like abundance patterns among metal-poor stars (such a large enrichment in $\mathrm{Al}$ has not been observed in dwarf galaxy stellar populations; see Shetrone et al. 2003; Hasselquist et al. 2017). Here, we relax this restriction in order to include those mildly metal-poor giants with moderate enrichment in $\mathrm{Al}$, as illustrated in Fig. 4.

With this large sample, we find that $\gtrsim 80 \%$ of the newly identified stars in the Jurassic structure display an aluminum 


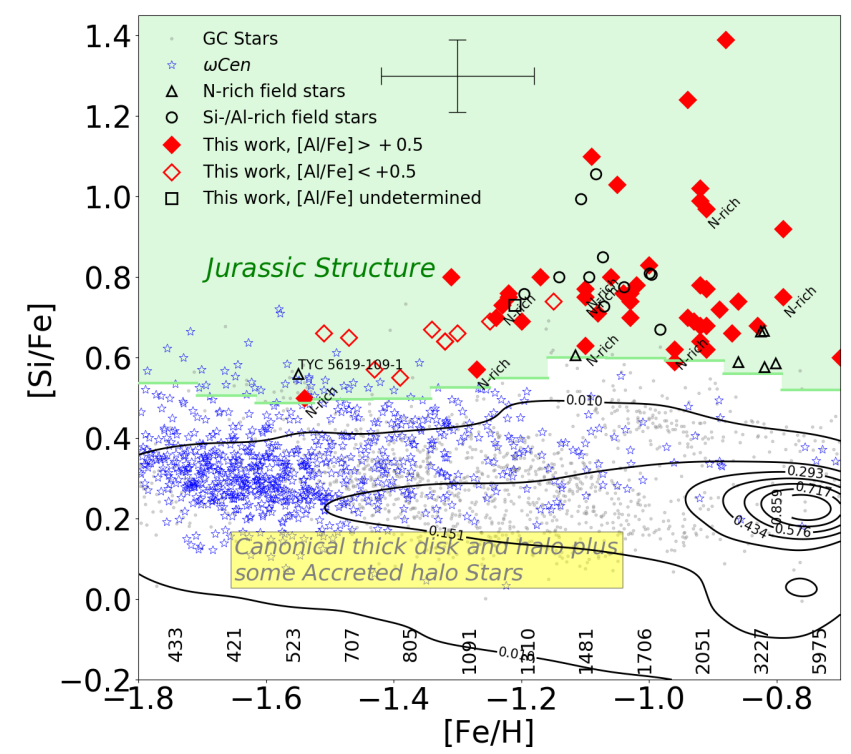

Fig. 3. Kernel Density Estimation (KDE) of the ASPCAP-derived [Si/Fe] abundances for MW stars, as a function of $[\mathrm{Fe} / \mathrm{H}]$, with contours showing the density of stars that appear to be a metal-poor extension of the thick disk and halo locus. These contours demonstrate that there is a low-density boundary (light green thick lines) separating two higher density regions, one with lower $[\mathrm{Si} / \mathrm{Fe}]$ (black contours), and one with higher $[\mathrm{Si} / \mathrm{Fe}]$ (light green shadow region). The BACCHUS-derived $[\mathrm{Si} / \mathrm{Fe}]$ abundance for the newly identified Si-enhanced stars is highlighted with a black empty square (undetermined $[\mathrm{Al} / \mathrm{Fe}]$ ), red empty diamonds $([\mathrm{Al} / \mathrm{Fe}] \lesssim+0.5$ in both the intermediate- and low-aluminum regimes, which are likely to be chemically tagged as escaped former members of MW dwarf spheroidal satellites or GCs), and red filled diamonds (with $[\mathrm{Al} / \mathrm{Fe}] \gtrsim+0.5$ or Al-enhanced stars, which are likely to be chemically tagged as migrants from GCs). Over-plotted are data from Fernández-Trincado et al. (2016b, 2017, 2019b; empty black triangles), from Fernández-Trincado et al. (2019c; empty black circles), and GC stars from Mészáros et al. (2020; gray dots and empty blue star symbols-highlighting the $\omega$ Centauri population). The plotted error bars show the median abundance uncertainty from BACCHUS. The number of APOGEE-2+ stars each bin are shown at the center of the bin at the bottom of the plot.

enrichment $([\mathrm{Al} / \mathrm{Fe}]>+0.5)$ above the Galactic levels, while $220 \%$ of stars in our sample lie in a group with $-0.1 \lesssim[\mathrm{Al} / \mathrm{Fe}] \lesssim+0.5$, which is likely part of the $[\mathrm{Al} / \mathrm{Fe}]-[\mathrm{Si} / \mathrm{Fe}]$ tail of the distribution of stars in the Jurassic structure, extending approximately from sub-Solar to super-Solar $[\mathrm{Al} / \mathrm{Fe}]$, as seen in Fig. 4.

As before, we ran a Kernel Density Estimation (KDE) model over the stars in the Jurassic structure, and compared them with the KDE distribution of the Si-normal giants belonging to the main body of the MW (see Fig. 4). The Jurassic structure is centred at around $\{[\mathrm{Al} / \mathrm{Fe}],[\mathrm{Si} / \mathrm{Fe}]\}=\{+0.75,+0.74\}$, while the $\mathrm{Si}$-normal (MW halo and disk system) stars run roughly between $\{[\mathrm{Al} / \mathrm{Fe}],[\mathrm{Si} / \mathrm{Fe}]\} \sim\{-0.26,+0.20\}$ and $\{+0.19,+0.25\}$. Nevertheless, close inspection of Fig. 4 reveals a fairly clear clump of giants (the Jurassic structure) which is not located on the main bulk of the KDE of the MW sample, and well-separated from the main body, exceeding the background level by a factor of 20-28 (five times more significant that determined previously). A set of white contour lines is provided as a visual aid. The [Si,Al/Fe]-peak $\gtrsim+0.5$ is clearly visible in Fig. 4 , which corresponds to the stars in the Jurassic structure of predominantly more metal-rich $([\mathrm{Fe} / \mathrm{H}] \gtrsim-1.3)$ stars enriched in $\mathrm{Si}$ and Al, compared to their extended tail, which extends from super-

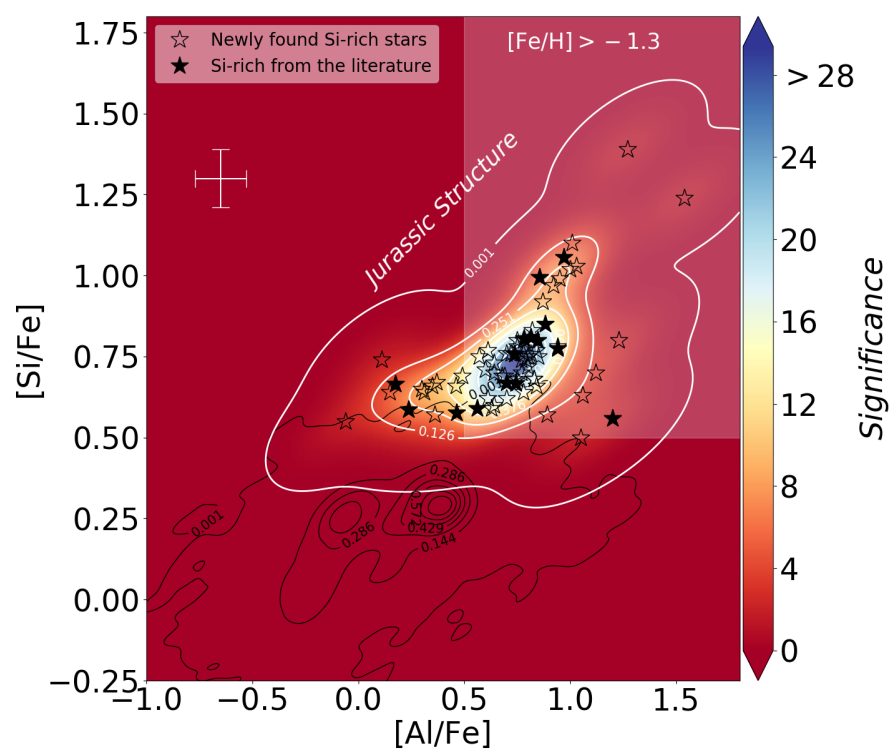

Fig. 4. Distribution of $[\mathrm{Si} / \mathrm{Fe}]$ vs. $[\mathrm{Al} / \mathrm{Fe}]$ for the APOGEE-2+ stars surviving the quality cuts discussed in Sect. 3. Isophotes corresponding to the chemical domain of the relatively more metal-poor thick disk and canonical and accreted halo populations are also superimposed, while individual stars are plotted where APOGEE-2+ observed stars are less populous in this plane.

Solar to sub-Solar $\mathrm{Al}([\mathrm{Al} / \mathrm{Fe}]<+0.5)$, and having $[\mathrm{Fe} / \mathrm{H}]<-1.3$ extending down to $[\mathrm{Fe} / \mathrm{H}] \sim-1.8$. The majority of stars in our final sample set lie in a group with super-Solar $[\mathrm{Al} / \mathrm{Fe}]$ and $[\mathrm{Si} / \mathrm{Fe}]$, which makes them unlikely to be field stars chemically tagged as migrants from dwarf galaxies. However, it is very likely that the parent systems were predominantly metal-rich, $[\mathrm{Fe} / \mathrm{H}]>-1.3$. This result confirms and reinforces the existence of a new stellar sub-population in the inner stellar halo of the MW, which is clearly well-separated from the normal halo and disk system.

\section{Elemental abundance analysis}

The results of the derived elemental abundances are shown in Figs. 5 and 6. For all the chemical species we have employed the BACCHUS code, and manually tweaked the $[\mathrm{X} / \mathrm{Fe}]$ and $[\mathrm{Fe} / \mathrm{H}]$ abundance of each line until the synthetic profile matched the observed profile. The results are compared to GC stars from Mészáros et al. (2020) spanning the same metallicity range as our sample.

\subsection{The iron-peak element: $F e$}

The Jurassic structure spans a wide range in metallicity, of $\sim 0.8 \mathrm{dex}$, with two apparent peaks at $[\mathrm{Fe} / \mathrm{H}]=-1.2$ and -0.9 , and an extended tail to lower metallicity $([\mathrm{Fe} / \mathrm{H}] \lesssim-1.5)$, which provides further evidence for several progenitors being responsible for producing the anomalous abundance ratios of [Si/Fe]. This could explain the disparate regions of the chemical space (see Fig. 5) and integrals of motion space (see Fig. 7) described below.

\subsection{The light-elements: $C$ and $N$}

Figure 5 show that carbon and nitrogen span similar large ranges in $[\mathrm{C} / \mathrm{Fe}]=\{-0.31,+0.67\}$ and $[\mathrm{N} / \mathrm{Fe}]=\{-0.11,+1.33\}$, 


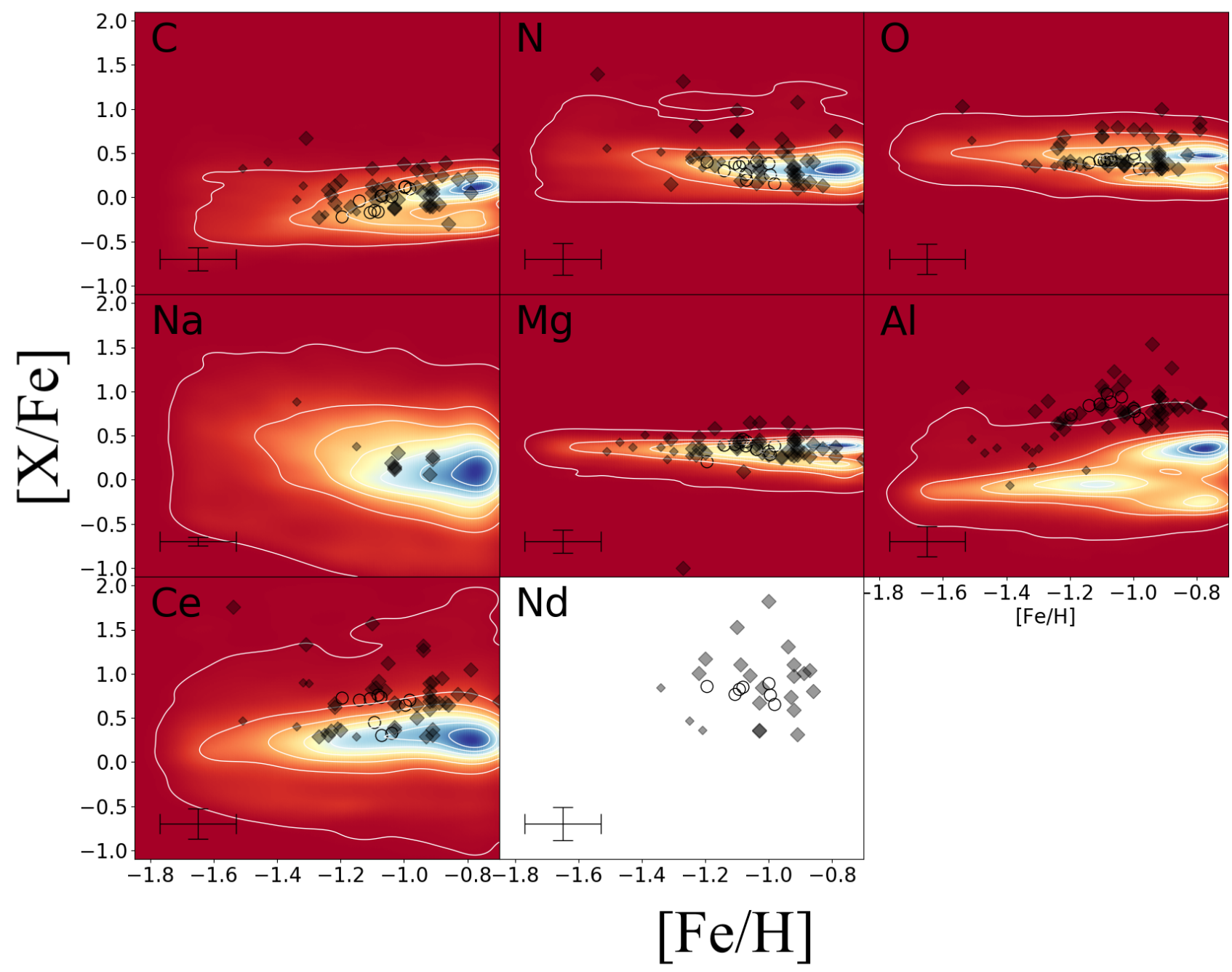

Fig. 5. Kernel Density Estimation (KDE) of $[\mathrm{X} / \mathrm{Fe}]$ with metallicity for the APOGEE-2+ stars surviving the quality cuts discussed in Sect. 3 . Stars in the Jurassic structure are shown as black diamonds. The small diamond symbols mark the Si-rich stars with $[\mathrm{Al} / \mathrm{Fe}]<+0.5$, while the large diamond symbols refer to Si-rich stars with $[\mathrm{Al} / \mathrm{Fe}] \gtrsim+0.5$. Previously discovered Si-rich stars from Fernández-Trincado et al. (2019c) are highlighted with black open circles.

with a few $(\sim 14 \%)$ stars being slightly enhanced in carbon $([\mathrm{C} / \mathrm{Fe}] \gtrsim+0.2)$, along with simultaneous enrichment in the $s$ process elements $([\mathrm{Ce}, \mathrm{Nd} / \mathrm{Fe}] \gtrsim+0.3,+1.3)$, contributing to the subclass of the carbon-enhanced metal-poor $s$-process-enriched (CEMP-s) stars at similar metallicity (Carollo et al. 2014; Beers et al. 2017), but displaying similar star-to-star scatter as that seen in Galactic GC stars at similar metallicity (Mészáros et al. 2015, 2020; Masseron et al. 2019). It is worth mentioning that a possible cause for the large dispersion observed in $[\mathrm{C} / \mathrm{Fe}]$ and $[\mathrm{N} / \mathrm{Fe}]$ could partially be attributed to the sensitivity of these elements to the atmospheric parameters. This could be due to the molecular equilibria that exist in the stellar atmosphere between ${ }^{16} \mathrm{OH},{ }^{12} \mathrm{C}^{16} \mathrm{O}$, and ${ }^{12} \mathrm{C}^{14} \mathrm{~N}$, as the strengths of the molecular features are strongly dependent on the surface temperature $\left(T_{\mathrm{eff}}\right)$ and gravity $(\log g)$, in particular for the warmer metal-poor giant stars.

\subsection{The $\alpha$-elements: $\mathrm{O}$ and $\mathrm{Mg}$}

Figure 5 show that our $[\mathrm{O} / \mathrm{Fe}]$ ratios exhibit evidence of an oxygen-poor $([\mathrm{O} / \mathrm{Fe}] \lesssim+0.5)$ and oxygen-rich $([\mathrm{O} / \mathrm{Fe}] \gtrsim+0.5)$ sequence in both the intermediate and low- $[\mathrm{Fe} / \mathrm{H}]$ regime, likely indicating a distinct formation history for each sequence. The stars in the Jurassic structure in the oxygen-poor sequence are slightly more enhanced than the canonical components of the MW, but both span similar ranges as that observed in GC stars at similar metallicity.

From Fig. 5, we also note that the peculiar Si-enhanced stars in GCs (Mészáros et al. 2020) occupy similar loci in [O/Fe] as those populated by our sample. The remaining $\alpha$-element abundance $(\mathrm{Mg})$ appears to be very mixed, making it difficult to disentangle a clear $\alpha$-poor sequence from an $\alpha$-rich one, which could be a result of the $\mathrm{O}$ and $\mathrm{Mg}$ SNII yields being mass dependent (see, e.g., Nomoto et al. 2013; Hawkins et al. 2015).

We also find a star (2M22375002-1654304) in the Jurassic structure whose chemistry is consistent with a genuine second-generation GCs. 2M22375002-1654304 is a not carbon-enhanced metal-poor star $([\mathrm{Fe} / \mathrm{H}] \sim-1.27)$ which has a $[\mathrm{Mg} / \mathrm{Fe}]$ ratio of $\sim-1$ accompained by a modest enrichment in $[\mathrm{N}, \mathrm{Al}, \mathrm{Si} / \mathrm{Fe}] \gtrsim+0.5$, which is the typical signature of secondgeneration GC stars (see, e.g., Pancino et al. 2017; Masseron et al. 2019; Mészáros et al. 2020). It is support with the hypothesis that the Jurassic structure could be made up of dissipated GCs. 

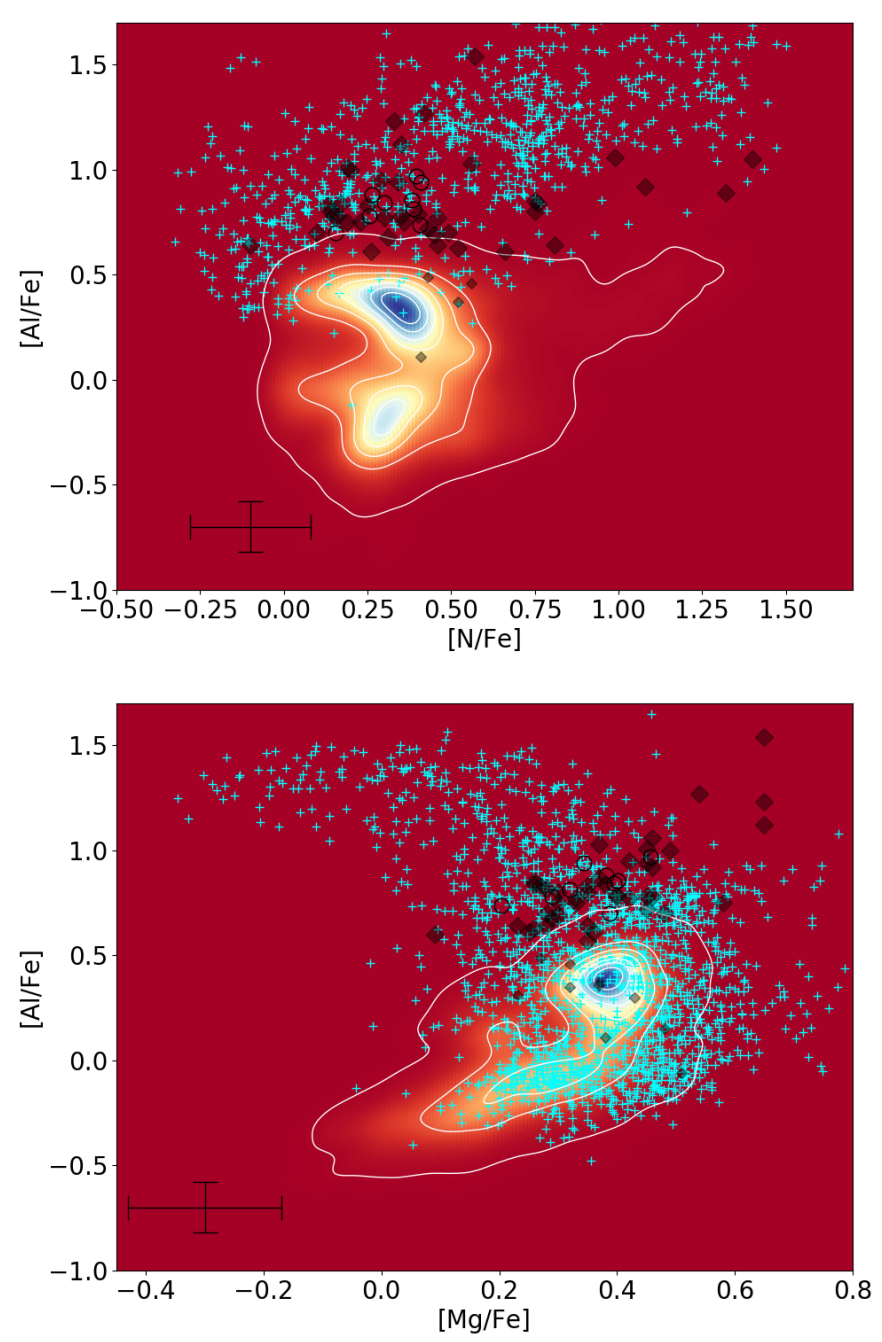

Fig. 6. Same as Fig. 5, but for abundance distributions of $[\mathrm{Al} / \mathrm{Fe}]$ with $[\mathrm{N} / \mathrm{Fe}]$ (top) and $[\mathrm{Mg} / \mathrm{Fe}]$ (bottom). Over-plotted (cyan crosses) are GCs from Mészáros et al. (2020) at similar metallicity as the Si-rich stars.

\subsection{The odd-Z elements: $\mathrm{Na}$ and $\mathrm{Al}$}

We find a noticeable trend of $[\mathrm{Al} / \mathrm{Fe}]$ with metallicity (see Fig. 3); the more metal-poor stars in our sample exhibit a low aluminum enrichment $([\mathrm{Al} / \mathrm{Fe}] \lesssim+0.5)$, making them equally probable to be associated with dwarf galaxy stars and/or Galactic GCs, while the more metal-rich $([\mathrm{Fe} / \mathrm{H}] \gtrsim-1.2)$ stars in our sample are generally more enriched in aluminum than typically seen in dwarf galaxy stars (see, e.g., Hasselquist et al. 2017, 2019; Hayes et al. 2018; Nidever et al. 2020). These stars are more likely linked to a $\mathrm{GC}$ origin, and span a similar $[\mathrm{Al} / \mathrm{Fe}]$ spread with an apparent weak N-Al correlation (see Fig. 6 top) as that observed in metal-rich/-poor GC stars (Mészáros et al. 2020).

From Fig. 6 bottom, we also note two trends of $[\mathrm{Al} / \mathrm{Fe}]$ with $[\mathrm{Mg} / \mathrm{Fe}]$; the stars in the Jurassic structure with low- $[\mathrm{Al} / \mathrm{Fe}]$ $([\mathrm{Al} / \mathrm{Fe}] \lesssim+0.5)$ stars follow an apparent $\mathrm{Al}-\mathrm{Mg}$ anti-correlation as that seen in Galactic GCs (Mészáros et al. 2020). However, an unexpected turnover in the Al-Mg diagram is also clearly seen in Fig. 3, where the aluminum-enriched stars in our sample $([\mathrm{Al} / \mathrm{Fe}] \gtrsim+0.5)$ display an apparent $\mathrm{Al}-\mathrm{Mg}$ correlation, with the apparent existence of an $\mathrm{Al}-\mathrm{Si}$ correlation from the data in Table A.1. This could be interpreted as a signature of ${ }^{28} \mathrm{Si}$ leakage from the $\mathrm{MgAl}$ chain (Masseron et al. 2019; Mészáros et al. 2020 ) in their different progenitors, likely happening toward higher metallicities $([\mathrm{Fe} / \mathrm{H}] \gtrsim-1.2)$. It is important to note, however, that the ${ }^{28} \mathrm{Si}$ leakage has been only observed thus far in metal-poor ([Fe/H] $\lesssim-1.2)$ GCs (see, e.g., Masseron et al. 2019; Mészáros et al. 2020), while the stars in the Jurassic structure are particularly more metal rich.

Whether some of the metal-rich stars in the Jurassic structure were part of the GC stars that have fully migrated into the inner halo of the MW could in part explain their lack in metal-rich GCs, but it is still unclear. However, our finding may indicate that the temperature conditions of the stars in the progenitors were too high to efficiently produce the ${ }^{28} \mathrm{Si}$ leakage from Mg-Al chain (Prantzos et al. 2017; Masseron et al. 2019) toward higher metallicities.

Figure 5 shows the trends of sodium as a function of metallicity for our sample. The abundance of Na exhibits a small dispersion $(<0.22$ dex $)$ and remains rather constant at $[\mathrm{Fe} / \mathrm{H}] \gtrsim-1.2$, except for one metal-poor star in our sample that exhibits a high enrichment in $[\mathrm{Na} / \mathrm{Fe}] \gtrsim+0.8$. However, it is important to note that $[\mathrm{Na} / \mathrm{Fe}]$ abundances have been found to be typically affected by large uncertainties ( $~ 20.2 \mathrm{dex})$, as its two lines $(1.6373 \mu \mathrm{m}$ and $1.6388 \mu \mathrm{m})$ in our APOGEE-2+ spectra are weak (line intensity is comparable to the variance) and possibly blended at the typical $T_{\text {eff }}$ and metallicity of our sample, which would lead to unreliable abundance results. For this reason, the $[\mathrm{Na} / \mathrm{Fe}]$ listed in Table A.2 are listed as upper limits.

\subsection{The s-process elements: $\mathrm{Ce}$ and $\mathrm{Nd}$}

The Jurassic structure exhibits a clear enhancement of the heavy second $s$-process-peak elements, such as $\mathrm{Ce}$ and $\mathrm{Nd}$, as shown in Fig. 5, with $[\mathrm{Ce} / \mathrm{Fe}]=+0.29$ to +1.76 and $[\mathrm{Nd} / \mathrm{Fe}]=+0.31$ to +1.82 . Depending on the chemical composition of the other chemical species, the stars in the Jurassic structure could owe itheir heavy-element abundance patterns to different possible channels, likely by contamination from mass transfer (see, e.g., Preston \& Sneden 2001; Lucatello et al. 2003; Sivarani et al. 2004; Barbuy et al. 2005; Thompson et al. 2008; Fernández-Trincado et al. 2019a), or by pollution of gas already strongly enriched in $s$-process elements.

The modest carbon enhancement observed in a handful ( $\sim 14 \%$ of the stars in our sample) of stars in the Jurassic structure may raise the possibility that other enrichment processes, rather than the mass-transfer hypothesis, could be responsible for the over-abundance in $s$-process elements. These abundance signatures are often observed among chemically peculiar giant stars, CEMP- $s$ stars, and low-/intermediate-mass asymptotic giant branch (AGB) stars at this metallicity (see, e.g., Carollo et al. 2014; Fernández-Trincado et al. 2016b; Ventura et al. 2016; Beers et al. 2017; Pereira et al. 2017, 2019), or by pollution of nearby massive $\left(10-300 M_{\odot}\right)$ stars (see, e.g., Masseron et al. 2020). Additionally, there is good agreement in the $[\mathrm{Ce}, \mathrm{Nd} / \mathrm{Fe}]$ abundance ratios derived in this work with the $s$-process elements in some GC stars (Masseron et al. 2019; Mészáros et al. 2020).

\section{Orbits}

We used the GravPot $16^{1}$ code to study the Galactic orbits of the stars in the Jurassic structure. For this purpose, we combine accurate proper motions from Gaia DR2 (Gaia Collaboration 2018; Lindegren et al. 2018), radial velocities from APOGEE-2+ (Nidever et al. 2015; Majewski et al. 2017;

\footnotetext{
https://gravpot.utinam.cnrs. fr
} 


\section{$\Omega_{b a r}=43 \mathrm{~km} \mathrm{~s}^{-1} \mathrm{kpc}^{-1}$}

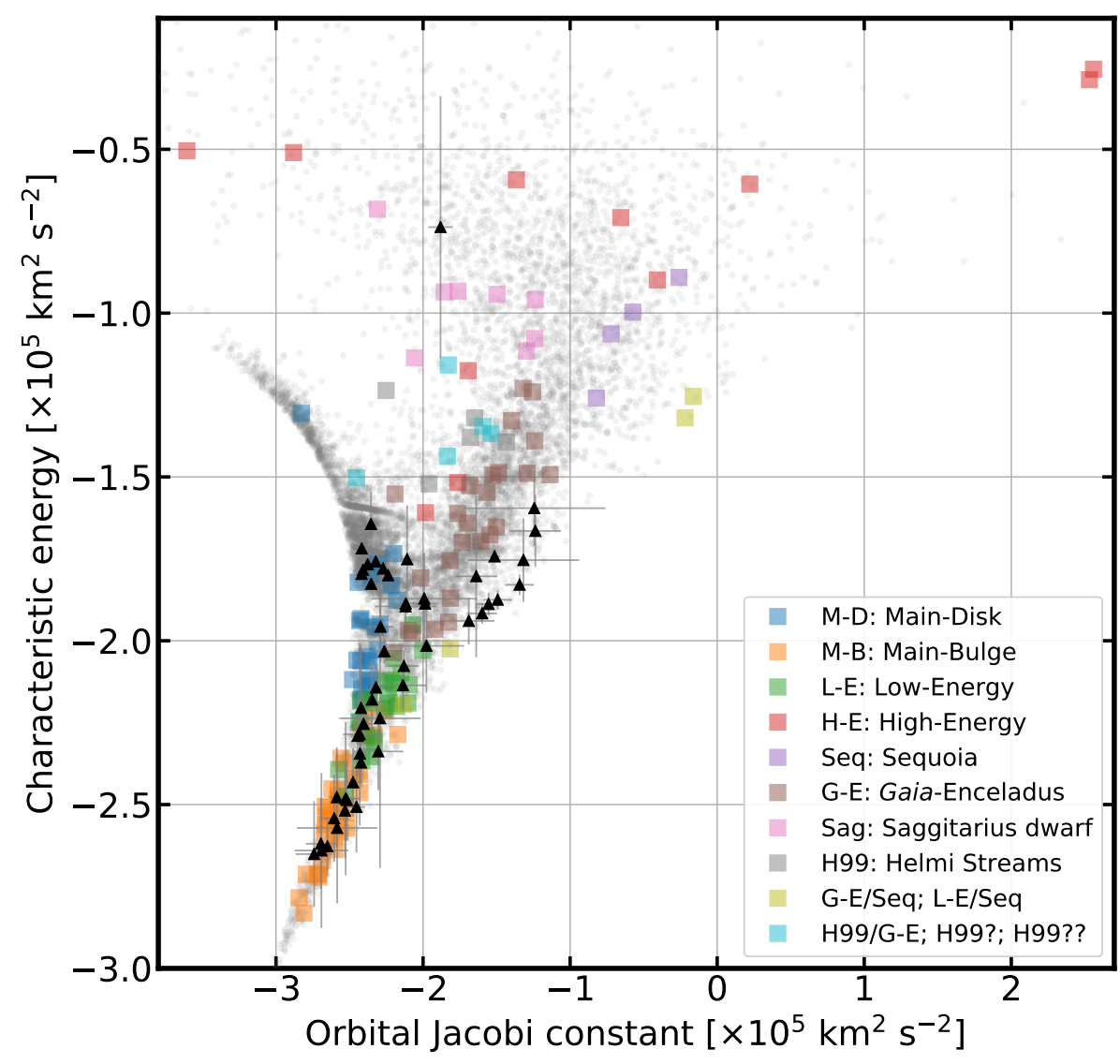

Fig. 7. Characteristic orbital energy $\left(\left(E_{\max }+\right.\right.$ $\left.E_{\min }\right) / 2$ ) versus the orbital Jacobi constant $\left(E_{\mathrm{J}}\right)$ in the non-inertial reference frame where the bar is at rest. Square symbols refer to Galactic GCs, color-coded according to their associations with different progenitors from Massari et al. (2019). The black triangles with error bars refer to the stars in the Jurassic structure analyzed in this study. The gray dots show the APOGEE-2+ stars surviving the quality cuts discussed in Sect. 3.
Ahumada et al. 2020), and distances from StarHorse (Anders et al. 2019; Queiroz et al. 2018, 2020a,b) as input data in our model. Only stars with a re-normalized unit weight error (Lindegren et al. 2018), RUWE $<1.4$ (stars with reliable proper motions) were considered in our orbital analysis.

The Galactic potential assumed in these calculations is the non-axisymmetric MW-like potential, which considers perturbations due to a rotating boxy/peanut bar. This potential fits the structural and dynamical parameters of the Galaxy, based on recent knowledge of our MW. For each star, we computed an ensemble of orbits by assuming three different values for the angular velocity of the bar, $\Omega_{\mathrm{bar}}=33,43$, and $53 \mathrm{~km} \mathrm{~s}^{-1} \mathrm{kpc}^{-1}$, with a bar mass of $1.1 \times 10^{10} M_{\odot}$, and a present-day angle orientation of $20^{\circ}$, in the same manner as in Fernández-Trincado et al. (2020a). To model the uncertainty distributions, we sampled one million orbits using a simple Monte Carlo approach assuming Gaussian distributions for the input parameters (heliocentric distances, radial velocities, and proper motions), with $1 \sigma$ equal to the errors of the input parameters as listed in Table A.1. The main orbital elements $\left(\left|Z_{\text {max }}\right|, r_{\text {peri }}, r_{\text {apo }}\right.$, eccentricity, and the $z$-component of the angular momentum in the inertial frame) are listed in Table A.4. The data presented in this table correspond to a backward time integration of $3 \mathrm{Gyr}$, with error bars computed as $\Delta=(84$ th percentile -16 th percentile $) / 2$, while the number inside parenthesis indicate the sensitivity in the orbital elements due to the variations of the angular velocity of the bar. This table also list the minimum and maximum variation of the $z$-component of the angular momentum in the inertial frame, $L_{z}$, since this quantity is not conserved in a model like GravPot 16, and we are interested in the variation of $L_{z}$ along the full integration time, allowing us to identify the orbital configuration of each star: prograde, retrograde, and $\mathrm{P}-\mathrm{R}^{2}$ Orbits are calculated with respect to the rotation of the bar.

For reference, the Galactic convention adopted by this work is: $X$-axis is oriented toward $l=0^{\circ}$ and $b=0^{\circ}$, and the $Y$ axis is oriented toward $l=90^{\circ}$ and $b=0^{\circ}$, and the disk rotates toward $l=90^{\circ}$; the velocities are also oriented in these directions. In this convention, the Sun's orbital velocity vector is $\left[U_{\odot}\right.$, $\left.V_{\odot}, W_{\odot}\right]=[11.1,12.24,7.25] \mathrm{km} \mathrm{s}^{-1}$ (Brunthaler et al. 2011). The model has been rescaled to the Sun's Galactocentric distance, $8 \mathrm{kpc}$, and a local rotation velocity of $V_{\mathrm{LSR}}=244.5 \mathrm{~km} \mathrm{~s}^{-1}$ (Fernández-Trincado et al. 2020a).

We find that most of the stars in the Jurassic structure span a large range in heliocentric distances, $3<d<12 \mathrm{kpc}$, are more likely found at intermediate to high latitudes $|b|>10^{\circ}$, with a few exceptions to the inner regions of the MW, and currently are located far from the peri-/apocentre of their orbits.

The orbital elements reveal that most $(\sim 70 \%)$ of the stars in the Jurassic structure have highly eccentric orbits $(e \gtrsim 0.7)$, covering a wide range of vertical excursions from the Galactic plane $(\$ 46 \mathrm{kpc})$. These appear to behave as halo-like orbits, some of which have mid-and off-plane orbits passing through the inner Galaxy. A small fraction of the stars in the Jurassic structure have orbits concentrated at small heights above the

2 A P-R orbit is defined as to the one that flip its sense from prograde to retrograde, or vice-versa, along its orbit. 
plane, with apocentric distances, $r_{\text {apo }}$, that vary between $4.5 \mathrm{kpc}$ to $13 \mathrm{kpc}$, and pericenter distances between $\sim 3 \mathrm{kpc}$ to $6 \mathrm{kpc}$ from the Galactic Centre, with almost circular prograde orbits, suggesting a possible dynamical association with the thick disk.

For comparison with our sample, we calculated the orbital solutions for Galactic GCs from Baumgardt et al. (2019), and adopted the progenitor classification as in Massari et al. (2019). Figure 7 shows the characteristic orbital energy $\left(\left(E_{\max }+E_{\min }\right) / 2\right)$ versus the orbital Jacobi constant $\left(E_{\mathrm{J}}\right)$ distribution of Galactic GCs and the stars in the Jurassic structure. This diagram clearly shows that the stars in the Jurassic structure populate a wide range of energies, similar to that of Galactic GCs with different origins, suggesting that the stars in the Jurassic structure are peculiar objects that appear not to have emerged from a single system.

Figure 8 show the distribution of stars in the Jurassic structure in the Toomre diagram and $V_{\phi}$ vs. $V_{\mathrm{R}}$ space. In these plane, we can see that the vast majority of the stars in the Jurassic structure stand out in their distribution of velocity components and are clearly distinct from the disk, with a few exceptions associated with stars having low orbital eccentricities as listed in Table A.4, which have been likely trapped in corotation resonance with the bar. The $V_{\phi}$ vs. $V_{\mathrm{R}}$ plane, reveals that some of the stars in the Jurassic structure are associated with the Gaia-Enceladus as also revealed by their orbital elements as seen in Fig. 7. It is likely that the Jurassic structure is made up of the cumulative effect of several tidally disrupted GCs, some of which belonged to the Gaia-Enceladus accretion event.

From the energy plane (see Fig. 7), we can clearly distinguish three possible groups among the stars in the Jurassic structure. The stars in this structure with $E_{\mathrm{J}} \lesssim-2 \times 10^{5} \mathrm{~km}^{2} \mathrm{~s}^{-2}$ populate the region dominated by in situ clusters belonging to the subgroup of GCs in the Main-Disk (M-D), Main-Bulge (M-B), and Low-Energy (L-E) group (see, e.g., Massari et al. 2019), while one star in the Jurassic structure has an $E_{\mathrm{J}} \lesssim-2 \times 10^{5} \mathrm{~km}^{2} \mathrm{~s}^{-2}$, occupying the region populated by GCs belonging the highenergy (H-E) group and those associated with the Sagittarius (Sgr) dwarf spheroidal galaxy, with some contamination by clusters associated with the GSE progenitor(s) (see, e.g., Belokurov et al. 2018; Massari et al. 2019).

Strikingly, we also find a clear substructure of 10 stars in the Jurassic structure with $E_{\mathrm{J}} \gtrsim-2 \times 10^{5} \mathrm{~km}^{2} \mathrm{~s}^{-2}$, which appear contained within the region dominated by the subgroup of GCs associated with GSE (see, e.g., Massari et al. 2019). This particular subgroup of stars in the Jurassic structure correspond mostly with the stars with a low aluminum enrichment ([Al/Fe] $\lesssim+0.5)$, with retrograde orbits, eccentricities ranging from $e \sim 0.47$ to 0.9 , vertical excursions from the Galactic plane from $1.56 \mathrm{kpc}$ to $7.5 \mathrm{kpc}, r_{\text {peri }}=0.7-3.13 \mathrm{kpc}$, and $r_{\text {apo }}=7.8-15.36 \mathrm{kpc}$. Thus, given the low $[\mathrm{Al} / \mathrm{Fe}]$ of some $(6$ out of 10) stars in this subgroup, they are likely associated with the accreted GSE progenitor(s) (e.g., Belokurov et al. 2018; Helmi et al. 2018; Haywood et al. 2018; Myeong et al. 2019), or possibly are linked to GC debris from the GSE merger event. For the remaining stars in the Jurassic structure with high-[Al/Fe] it is unlikely that they came from dwarf galaxy progenitors, as present-day stars in dwarf galaxy satellites do not exhibit $[\mathrm{Al} / \mathrm{Fe}]$ abundance ratios which exceed $\gtrsim+0.5$, thus the more probable origin of those stars may be associated with in situ and LowEnergy Galactic GCs, with a few exceptions, as the case of the stars in the Jurassic structure with orbital energies in the domain of the Sgr clusters (see Fig. 7).
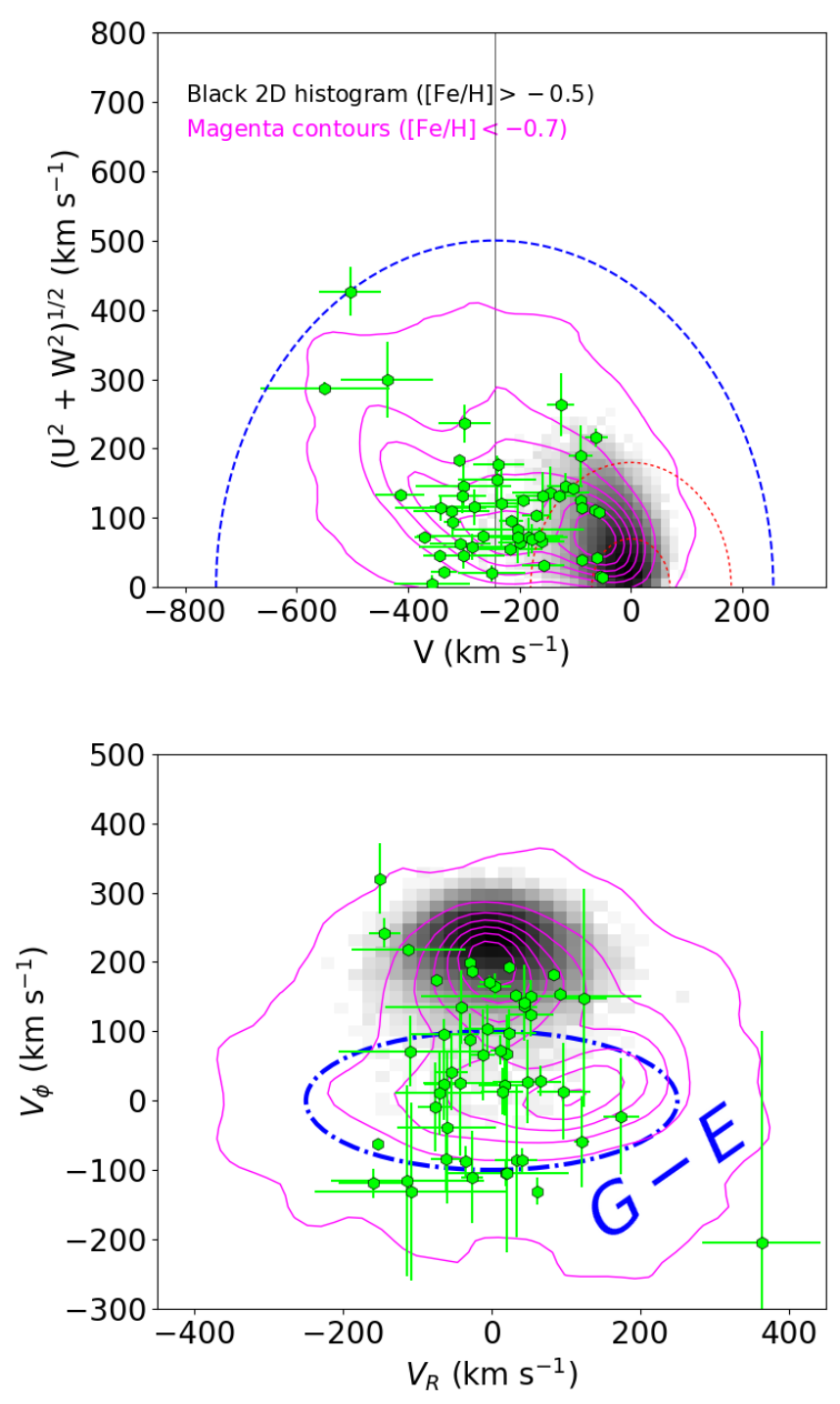

Fig. 8. Toomre diagram (top) for the stars in the Jurassic structure (lime symbols) compared to a control sample of 200000 APOGEE$2+$ stars with $[\mathrm{Fe} / \mathrm{H}]>-0.5$ and $d_{\odot}<5 \mathrm{kpc}$, likely associated with the thin and thick disk (2D histrogram), and a sample of $\sim 11000$ metalpoor stars, $[\mathrm{Fe} / \mathrm{H}]<-0.5$, likely associated with the halo and thick disk population (magenta contours). All velocities are relative to the LSR. The red dashed rings show roughly the boundaries of the thin and thick disk at a constant velocity of $\sim 70 \mathrm{~km} \mathrm{~s}^{-1}$ and $180 \mathrm{~km} \mathrm{~s}^{-1}$, respectively (Venn et al. 2004). The blue dashed ring represents a constant galacticrest frame velocity of $\sim 500 \mathrm{~km} \mathrm{~s}^{-1}$ shifted relative to the other velocities. The distribution of the velocity components $V_{\phi}$ vs. $V_{\mathrm{R}}$ (bottom) is also shown for our sample. The blue dashed ellipse represents the approximate region for stars associated with the Gaia-Enceladus in $V_{\phi}$ vs. $V_{\mathrm{R}}$ space based on Belokurov et al. (2018).

\section{Concluding remarks}

Using the internal APOGEE-2+ data set, we confirm and reinforce the existence of a distinct stellar population in the inner halo of the MW-the Jurassic structure. Here, we report on the identification of 55 new Si-rich, mildly metal-poor stars, 45 of which exhibit high $[\mathrm{Al} / \mathrm{Fe}] \gtrsim+0.5$, making it unlikely that dwarf galaxy satellites could have contributed the majority of these stars. We find some dynamical evidencethat a few of them, in particular the stars in the Jurassic structure with $[\mathrm{Al} / \mathrm{Fe}] \lesssim+0.5$, could be associated with the accreted GSE dwarf galaxy and/or GCs of the GSE progenitor(s). 
We also find that the large majority of the new stars in the Jurassic structure exhibit chemical and dynamical properties similar to that of in situ GCs, supporting the picture that these peculiar stars have likely been dynamically ejected into the inner halo by dissolved GCs. This discovery could aid in explaining the assembly of the mildly metal-poor $([\mathrm{Fe} / \mathrm{H}] \sim-1)$ component of the inner Galactic halo and its complex formation process.

Acknowledgements. The author is grateful for the enlightening feedback from the anonymous referee. J.G.F.-T. is supported by FONDECYT No. 3180210 T.C.B. acknowledges partial support for this work from grant PHY 14-30152: Physics Frontier Center/JINA Center for the Evolution of the Elements (JINA CEE), awarded by the US National Science Foundation. D.M. is supported by the BASAL Center for Astrophysics and Associated Technologies (CATA) through grant AFB 170002, and by project FONDECYT Regular No. 1170121. This work has made use of data from the European Space Agency (ESA) mission Gaia (http://www. cosmos. esa.int/gaia), processed by the Gaia Data Processing and Analysis Consortium (DPAC, http: //www . cosmos. esa . int/ web/gaia/dpac/consortium). Funding for the DPAC has been provided by national institutions, in particular the institutions participating in the Gaia Multilateral Agreement. Funding for the Sloan Digital Sky Survey IV has been provided by the Alfred P. Sloan Foundation, the US Department of Energy Office of Science, and the Participating Institutions. SDSS- IV acknowledges support and resources from the Center for High-Performance Computing at the University of Utah. The SDSS website is www.sdss.org. SDSS-IV is managed by the Astrophysical Research Consortium for the Participating Institutions of the SDSS Collaboration including the Brazilian Participation Group, the Carnegie Institution for Science, Carnegie Mellon University, the Chilean Participation Group the French Participation Group, Harvard-Smithsonian Center for Astrophysics, Instituto de Astrofisica de Canarias, The Johns Hopkins University, Kavli Institute for the Physics and Mathematics of the Universe (IPMU)/University of Tokyo, Lawrence Berkeley National Laboratory, Leibniz Institut für Astrophysik Potsdam (AIP), Max-Planck-Institut für Astronomie (MPIA Heidelberg), Max-Planck-Institut für Astrophysik (MPA Garching), Max-Planck-Institut für Extraterrestrische Physik (MPE), National Astronomical Observatory of China, New Mexico State University, New York University, University of Notre Dame, Observatório Nacional/MCTI, The Ohio State University, Pennsylvania State University, Shanghai Astronomical Observatory, United Kingdom Participation Group, Universidad Nacional Autónoma de México, University of Arizona, University of Colorado Boulder, University of Oxford, University of Portsmouth University of Utah, University of Virginia, University of Washington, University of Wisconsin, Vanderbilt University, and Yale University.

\section{References}

Ahumada, R., Prieto, C. A., Almeida, A., et al. 2020, ApJS, 249, 3 Alvarez, R., \& Plez, B. 1998, A\&A, 330, 1109

An, D., \& Beers, T. C. 2020, ApJ, 897, 39

An, D., Beers, T. C., Johnson, J. A., et al. 2013, ApJ, 763, 65

An, D., Beers, T. C., Santucci, R. M., et al. 2015, ApJ, 813, L28

Anders, F., Khalatyan, A., Chiappini, C., et al. 2019, A\&A, 628, A94

Asplund, M., Grevesse, N., \& Sauval, A. J. 2005, in Cosmic Abundances as

Records of Stellar Evolution and Nucleosynthesis, eds. T. G. Barnes, III, \& F

N. Bash, ASP Conf. Ser., 336, 25

Barbuy, B., Spite, M., Spite, F., et al. 2005, A\&A, 429, 1031

Bastian, N., \& Lardo, C. 2018, ARA\&A, 56, 83

Baumgardt, H., Hilker, M., Sollima, A., \& Bellini, A. 2019, MNRAS, 482, 5138

Beers, T. C., \& Christlieb, N. 2005, ARA\&A, 43, 531

Beers, T. C., Carollo, D., Ivezić, Ž., et al. 2012, ApJ, 746, 34

Beers, T. C., Placco, V. M., Carollo, D., et al. 2017, ApJ, 835, 81

Bekki, K. 2019, MNRAS, 490, 4007

Belokurov, V., Erkal, D., Evans, N. W., Koposov, S. E., \& Deason, A. J. 2018, MNRAS, 478, 611

Blanton, M. R., Bershady, M. A., Abolfathi, B., et al. 2017, AJ, 154, 28

Bowen, I. S., \& Vaughan, A. H. 1973, Appl. Opt., 12, 1430

Bressan, A., Marigo, P., Girardi, L., et al. 2012, MNRAS, 427, 127

Brunthaler, A., Reid, M. J., Menten, K. M., et al. 2011, Astron. Nachr., 332, 461

Carollo, D., Beers, T. C., Lee, Y. S., et al. 2007, Nature, 450, 1020

Carollo, D., Beers, T. C., Chiba, M., et al. 2010, ApJ, 712, 692

Carollo, D., Martell, S. L., Beers, T. C., \& Freeman, K. C. 2013, ApJ, 769, 87

Carollo, D., Freeman, K., Beers, T. C., et al. 2014, ApJ, 788, 180

Chiba, M., \& Beers, T. C. 2000, AJ, 119, 2843

Cunha, K., Smith, V. V., Hasselquist, S., et al. 2017, ApJ, 844, 145

Eisenstein, D. J., Weinberg, D. H., Agol, E., et al. 2011, AJ, 142, 72

Feltzing, S., \& Chiba, M. 2013, New Astron. Rev., 57, 80
Fernández-Alvar, E., Carigi, L., Schuster, W. J., et al. 2018, ApJ, 852, 50

Fernández-Alvar, E., Fernández-Trincado, J. G., Moreno, E., et al. 2019, MNRAS, 487, 1462

Fernández-Trincado, J. G., Vivas, A. K., Mateu, C. E., \& Zinn, R. 2013, Mem. Soc. Astron. It., 84, 265

Fernández-Trincado, J. G., Vivas, A. K., Mateu, C. E., et al. 2015a, A\&A, 574, A15

Fernández-Trincado, J. G., Robin, A. C., Vieira, K., et al. 2015b, A\&A, 583, A76 Fernández-Trincado, J. G., Robin, A. C., Reylé, C., et al. 2016a, MNRAS, 461, 1404

Fernández-Trincado, J. G., Robin, A. C., Moreno, E., et al. 2016b, ApJ, 833, 132 Fernández-Trincado, J. G., Zamora, O., García-Hernández, D. A., et al. 2017, ApJ, 846, L2

Fernández-Trincado, J. G., Mennickent, R., Cabezas, M., et al. 2019a, A\&A, 631, A97

Fernández-Trincado, J. G., Beers, T. C., Tang, B., et al. 2019b, MNRAS, 488, 2864

Fernández-Trincado, J. G., Beers, T. C., Placco, V. M., et al. 2019c, ApJ, 886, L8 Fernández-Trincado, J. G., Zamora, O., Souto, D., et al. 2019d, A\&A, 627, A178 Fernández-Trincado, J. G., Chaves-Velasquez, L., Pérez-Villegas, A., et al. 2020a, MNRAS, 495, 4113

Fernández-Trincado, J. G., Beers, T. C., Minniti, D., et al. 2020b, A\&A, 643, L4 Fernández-Trincado, J. G., Beers, T. C., Minniti, D., et al. 2020c, ApJ, 903, L17 Fernández-Trincado, J. G., Minniti, D., Beers, T. C., et al. 2020d, A\&A, 643, A145

Freeman, K., \& Bland-Hawthorn, J. 2002, ARA\&A, 40, 487

Gaia Collaboration (Brown, A. G. A., et al.) 2018, A\&A, 616, A1

García Pérez, A. E., Allende Prieto, C., Holtzman, J. A., et al. 2016, AJ, 151, 144

González Hernández, J. I., \& Bonifacio, P. 2009, A\&A, 497, 497

Grevesse, N., Scott, P., Asplund, M., \& Sauval, A. J. 2015, A\&A, 573, A27

Gunn, J. E., Siegmund, W. A., Mannery, E. J., et al. 2006, AJ, 131, 2332

Gustafsson, B., Edvardsson, B., Eriksson, K., et al. 2008, A\&A, 486, 951

Hanke, M., Koch, A., Prudil, Z., Grebel, E. K., \& Bastian, U. 2020, A\&A, 637, A98

Hasselquist, S., Shetrone, M., Cunha, K., et al. 2016, ApJ, 833, 81

Hasselquist, S., Shetrone, M., Smith, V., et al. 2017, ApJ, 845, 162

Hasselquist, S., Carlin, J. L., Holtzman, J. A., et al. 2019, ApJ, 872, 58

Hawkins, K., Jofré, P., Masseron, T., \& Gilmore, G. 2015, MNRAS, 453, 758

Hawkins, K., Masseron, T., Jofré, P., et al. 2016, A\&A, 594, A43

Hayes, C. R., Majewski, S. R., Shetrone, M., et al. 2018, ApJ, 852, 49

Hayes, C. R., Majewski, S. R., Hasselquist, S., et al. 2020, ApJ, 889, 63

Haywood, M., Di Matteo, P., Lehnert, M. D., et al. 2018, ApJ, 863, 113

Helmi, A. 2020, ARA\&A, 58, 205

Helmi, A., White, S. D. M., de Zeeuw, P. T., \& Zhao, H. 1999, Nature, 402, 53

Helmi, A., Babusiaux, C., Koppelman, H. H., et al. 2018, Nature, 563, 85

Hogg, D. W., Casey, A. R., Ness, M., et al. 2016, ApJ, 833, 262

Holtzman, J. A., Shetrone, M., Johnson, J. A., et al. 2015, AJ, 150, 148

Holtzman, J. A., Hasselquist, S., Shetrone, M., et al. 2018, AJ, 156, 125

Ibata, R. A., Gilmore, G., \& Irwin, M. J. 1994, Nature, 370, 194

Ibata, R. A., Bellazzini, M., Malhan, K., Martin, N., \& Bianchini, P. 2019, Nat. Astron., 3, 667

Ivezić, Ž., Beers, T. C., \& Jurić, M. 2012, ARA\&A, 50, 251

Jönsson, H., Allende Prieto, C., Holtzman, J. A., et al. 2018, AJ, 156, 126

Jönsson, H., Holtzman, J. A., Prieto, C. A., et al. 2020, AJ, 160, 120

Koch, A., Grebel, E. K., \& Martell, S. L. 2019, A\&A, 625, A75

Koppelman, H., Helmi, A., \& Veljanoski, J. 2018, ApJ, 860, L1

Koppelman, H. H., Helmi, A., Massari, D., Roelenga, S., \& Bastian, U. 2019a, A\&A, 625, A5

Koppelman, H. H., Helmi, A., Massari, D., Price-Whelan, A. M., \& Starkenburg, T. K. 2019b, A\&A, 631, L9

Kruijssen, J. M. D., Pfeffer, J. L., Reina-Campos, M., Crain, R. A., \& Bastian, N. 2019, MNRAS, 486, 3180

Law, D. R., \& Majewski, S. R. 2010, ApJ, 718, 1128

Lind, K., Koposov, S. E., Battistini, C., et al. 2015, A\&A, 575, L12

Lindegren, L., Hernández, J., Bombrun, A., et al. 2018, A\&A, 616, A2

Lucatello, S., Gratton, R., Cohen, J. G., et al. 2003, AJ, 125, 875

Majewski, S. R., Zasowski, G., \& Nidever, D. L. 2011, ApJ, 739, 25

Majewski, S. R., Hasselquist, S., Łokas, E. L., et al. 2013, ApJ, 777, L13

Majewski, S. R., Schiavon, R. P., Frinchaboy, P. M., et al. 2017, AJ, 154, 94

Martell, S. L., Shetrone, M. D., Lucatello, S., et al. 2016, ApJ, 825, 146

Massari, D., Koppelman, H. H., \& Helmi, A. 2019, A\&A, 630, L4

Masseron, T., Merle, T., \& Hawkins, K. 2016, Astrophysics Source Code Library [record ascl:1605.004]

Masseron, T., García-Hernández, D. A., Mészáros, S., et al. 2019, A\&A, 622, A191

Masseron, T., García-Hernández, D. A., Santoveña, R., et al. 2020, Nat. Commun., 11, 3759 
Mészáros, S., Martell, S. L., Shetrone, M., et al. 2015, AJ, 149, 153

Mészáros, S., Masseron, T., García-Hernández, D. A., et al. 2020, MNRAS, 492, 1641

Myeong, G. C., Evans, N. W., Belokurov, V., Sanders, J. L., \& Koposov, S. E. 2018, ApJ, 863, L28

Myeong, G. C., Vasiliev, E., Iorio, G., Evans, N. W., \& Belokurov, V. 2019, MNRAS, 488, 1235

Naidu, R. P., Conroy, C., Bonaca, A., et al. 2020, ApJ, 901, 48

Nataf, D. M., Wyse, R. F. G., Schiavon, R. P., et al. 2019, AJ, 158, 14

Nidever, D. L., Holtzman, J. A., Allende Prieto, C., et al. 2015, AJ, 150, 173

Nidever, D. L., Hasselquist, S., Hayes, C. R., et al. 2020, ApJ, 895, 88

Nissen, P. E., \& Schuster, W. J. 2010, A\&A, 511, L10

Nomoto, K., Kobayashi, C., \& Tominaga, N. 2013, ARA\&A, 51, 457

Pancino, E., Romano, D., Tang, B., et al. 2017, A\&A, 601, A112

Pereira, C. B., Smith, V. V., Drake, N. A., et al. 2017, MNRAS, 469, 774

Pereira, C. B., Drake, N. A., \& Roig, F. 2019, MNRAS, 488, 482

Plez, B. 2012, Astrophysics Source Code Library [record ascl:1205.004]

Prantzos, N., Charbonnel, C., \& Iliadis, C. 2017, A\&A, 608, A28

Preston, G. W., \& Sneden, C. 2001, AJ, 122, 1545

Queiroz, A. B. A., Anders, F., Santiago, B. X., et al. 2018, MNRAS, 476, 2556

Queiroz, A. B. A., Chiappini, C., Perez-Villegas, A., et al. 2020a, A\&A, submitted [arXiv:2007.12915]

Queiroz, A. B. A., Anders, F., Chiappini, C., et al. 2020b, A\&A, 638, A76

Recio-Blanco, A., Rojas-Arriagada, A., de Laverny, P., et al. 2017, A\&A, 602, L14

Schiavon, R. P., Zamora, O., Carrera, R., et al. 2017, MNRAS, 465, 501

Shetrone, M., Venn, K. A., Tolstoy, E., et al. 2003, AJ, 125, 684

Shetrone, M., Bizyaev, D., Lawler, J. E., et al. 2015, ApJS, 221, 24

Simpson, J. D., Martell, S. L., Da Costa, G., et al. 2020, MNRAS, 491, 3374

Sivarani, T., Bonifacio, P., Molaro, P., et al. 2004, A\&A, 413, 1073

Smith, V. V., Cunha, K., Shetrone, M. D., et al. 2013, ApJ, 765, 16

Thomas, G. F., Jensen, J., McConnachie, A., et al. 2020, ApJ, 902, 89

Thompson, I. B., Ivans, I. I., Bisterzo, S., et al. 2008, ApJ, 677, 556
Ting, Y.-S., Conroy, C., \& Goodman, A. 2015, ApJ, 807, 104

Tissera, P. B., Beers, T. C., Carollo, D., \& Scannapieco, C. 2014, MNRAS, 439, 3128

Venn, K. A., Irwin, M., Shetrone, M. D., et al. 2004, AJ, 128, 1177

Ventura, P., García-Hernández, D. A., Dell'Agli, F., et al. 2016, ApJ, 831, L17

Wan, Z., Lewis, G. F., Li, T. S., et al. 2020, Nature, 583, 768

Wilson, J. C., Hearty, F., Skrutskie, M. F., et al. 2012, in Ground-based and Airborne Instrumentation for Astronomy IV, Proc. SPIE, 8446, 84460H

Wilson, J. C., Hearty, F. R., Skrutskie, M. F., et al. 2019, PASP, 131, 055001

Yuan, Z., Myeong, G. C., Beers, T. C., et al. 2020, ApJ, 891, 39

Zasowski, G., Johnson, J. A., Frinchaboy, P. M., et al. 2013, AJ, 146, 81

Zasowski, G., Cohen, R. E., Chojnowski, S. D., et al. 2017, AJ, 154, 198

\section{Appendix A: Tables}

We provide our results in four tables available at the CDS. The first table, a sample of which can be found in Table A.1, contains the basic parameters of stars in the Jurassic structure, i.e., 2MASS magnitudes, atmospheric parameters and radial velocity from the APOGEE2+ survey, astrometric information from Gaia DR2. Table A.2, contains the final abundances for every element (C, N, O, Na, Mg, Al, Si, Fe, Ce, and $\mathrm{Nd}$ ) and star on a line-by-line basis as analyzed in this work with the BACCHUS code (see Sect. 4 for more details), while the Table A.3, contains the uncertainties in the abundance caused by uncertainties in the stellar parameters for a small fraction of stars in our sample.

Table A.4, contains the main orbital elements of stars in the Jurassic structure predicted with the GravPot16 code (see Sect. 8 for more details). 